\title{
Decentralised control for complex systems - an invited survey
}

\section{Xing-Gang Yan*}

Instrumentation, Control and Embedded Systems Research Group,

School of Engineering and Digital Arts,

University of Kent,

Canterbury, Kent CT2 7NT, UK

E-mail: x.yan@kent.ac.uk

*Corresponding author

\section{Qingling Zhang}

Institute of Systems Science,

Northeastern University,

Shenyang, Liaoning, 110004, China

E-mail: qlzhang@mail.neu.edu.cn

\section{Sarah K. Spurgeon}

Instrumentation, Control and Embedded Systems Research Group,

School of Engineering and Digital Arts,

University of Kent,

Canterbury, Kent CT2 7NT, UK

E-mail: S.K.Spurgeon@kent.ac.uk

\section{Quanmin Zhu}

Department of Engineering Design and Mathematics,

University of the West of England,

Frenchy Campus,

Coldharbour Lane,

Bristol BS16 1QY, UK

E-mail: Quan.Zhu@uwe.ac.uk

\section{Leonid M. Fridman}

Departamento de Ingeniería de Control y Robótica,

Facultad de Ingeniería UNAM,

Mexico City 07000, Mexico

E-mail: 1fridman@unam.mx

\begin{abstract}
With the advancement of science and technology, practical systems are becoming more complex. Decentralised control has been recognised as a practical, feasible and powerful tool for application to large scale interconnected systems. In this paper, past and recent results relating to decentralised control of complex large scale interconnected systems are reviewed. Decentralised control based on modern control approaches such as variable structure techniques, adaptive control and backstepping approaches are discussed. It is well known that system structure can be employed to reduce conservatism in the control design and decentralised control for interconnected systems with similar and symmetric structure is explored. Decentralised control of singular large scale systems is also reviewed in this paper.
\end{abstract}

Keywords: large scale interconnected systems; decentralised control; singular systems; system structure.

Reference to this paper should be made as follows: Yan, X-G., Zhang, Q., Spurgeon, S.K., Zhu, Q. and Fridman, L.M. (2014) 'Decentralised control for complex systems - an invited survey', Int. J. Modelling, Identification and Control, Vol. 22, No. 4, pp.285-297.

Biographical notes: Xing-Gang Yan received his BSc from Shaanxi Normal University in 1985, MSc from Qufu Normal University in 1991, and $\mathrm{PhD}$ in Engineering from Northeastern University, China in 1997. Currently, he is a Lecturer at the University of Kent, UK. He was a Lecturer at Qingdao University, China from 1991 to 1994. He worked 
as a Research Fellow/Research Associate in the University of Hong Kong, China, Nanyang Technological University, Singapore and the University of Leicester, UK. He is a member of the Editorial Board of the Mathematical Problem of Engineering, International Journal of Engineering, and the Journal of Control Engineering and Technology. His research interests include sliding mode control, decentralised control, fault detection and isolation, control and observation of nonlinear systems and time delay systems with applications.

Qingling Zhang received his BSc and MSc from the Mathematics Department and PhD from the Automatic Control Department of Northeastern University, Shenyang, China in 1982, 1986 and 1995, respectively. He is Professor and Director of the Institute of Systems Science, Northeastern University. He has published 11 books and more than 600 papers in control theory and applications. He received the Golden Scholarship from Australia in 2000, and 14 prizes from central and local governments. He has visited the University of Kent, Hong Kong University, Sydney University, Western Australia University, Niigata University, Pohang University of Science and Technology, Seoul University, Alberta University, Lakehead University and Windsor University as a researcher or Visiting Professor. He is the Chairman of Liaoning Association of Mathematics and Asian Editor of Int. J. Information and Systems Science. His research includes bio-modelling, differential and algebraic systems and networked control systems.

Sarah K. Spurgeon is the Professor of Control Engineering and Head of the School of Engineering and Digital Arts at the University of Kent. Her research interests are in the area of systems modelling and analysis, robust control and estimation in which areas she has published over 270 refereed research papers. She was awarded the Honeywell International Medal for 'Distinguished contribution as a control and measurement technologist to developing the theory of control' in 2010 and an IEEE Millenium Medal in 2000. She is currently an IEEE Distinguished Lecturer and Chairs the IEEE Technical Committee on Variable Structure and Sliding Mode Control.

Quanmin Zhu received his MS in Harbin Institute of Technology, China in 1983 and PhD in Faculty of Engineering, University of Warwick, UK in 1989. Currently, he is a Professor in Control Systems at Department of Engineering Design and Mathematics, University of the West of England, Bristol, UK. His main research interest is in the area of nonlinear system modelling, identification, and control.

Leonid M. Fridman is a Professor in the Department of Control Engineering and Robotics at National Autonomous University of Mexico (UNAM), Mexico. His interest is variable structure systems. He is author and editor of seven books and 12 special issues on sliding mode control. $\mathrm{He}$ is a winner of Scopus Prize for the best cited Mexican Scientists in Mathematics and Engineering 2010. He was working as an Invited Professor in 19 universities and research centres of Argentina, Australia, France, China, Germany, Italy, Israel, and Spain.

\section{Introduction}

In the real world, there are a number of systems which can be modelled as dynamical equations composed of interconnections between a collection of lower-dimensional subsystems. Such classes of systems are called large-scale interconnected systems, which are often widely distributed in space (Lunze, 1992; Mahmoud, 2011; Sandell et al., 1978). A fundamental property of an interconnected system is that a perturbation of one subsystem can affect the other subsystems as well as the overall performance of the entire network. The purpose of control and monitoring paradigms from the domain of engineering within a large scale interconnected systems architecture is thus to minimise the effect of any perturbation or uncertainty on the overall system behaviour.

Large scale interconnected systems widely exist in society.A typical large scale interconnected system is the multi-machine power system (Wang et al., 1997; Yan et al., 2004). Other examples of large scale interconnected systems that present a great challenge to both system analysts and control designers include power networks, ecological systems, biological systems and energy systems (Mahmoud, 2011; Siljak, 1991).

For interconnected systems, the presupposition of centrality fails to hold due to either the lack of centralised information or the lack of centralised computing capability. When the number of subsystems is large, the computation time increases significantly if centralised control is employed. In the extreme case when information transfer among the subsystems is blocked, centralised control schemes simply cannot be applied. Even with engineered systems, issues such as the economic cost and reliability of communication links, particularly when systems are characterised by geographical separation, limit the appetite to develop centralised systems. From the perspective of economics and reliability, decentralised strategies are very 
pertinent for large scale interconnected systems. This has motivated the application of decentralised control methodologies to interconnected systems (Xie and Xie, 2000; Jiang, 2002; Liu et al., 2007; Panagi and Polycarpou, 2011). In Yang et al. (1998), a decentralised load-frequency controller design problem is translated into an equivalent problem of decentralised control design for a multi-input multi-output (MIMO) control system. A recent survey paper (Bakule, 2008) has covered several decomposition approaches such as disjoint subsystems, overlapping subsystems, symmetric composite systems, multi-time scale systems and hierarchically structured systems to simplify the analysis and synthesis tasks for large-scale systems to reduce the computation complexity.

Decentralised control for large-scale interconnected systems has been studied extensively. The application of decentralised schemes has dominated practice in industry due to the advantages of flexibility in operation, tolerance of failure and simplified design and tuning (Garelli et al., 2006). Research on large-scale interconnected systems analysis and synthesis can be traced back to at least the 1970s, and the survey paper (Sandell et al., 1978) clearly shows the development of this topic at that time when almost all of the work was focused on linear cases. With the advancement of technology and increasing requirements for high levels of performance, specifically in recent years, the dynamic systems used to model reality have become more complex involving nonlinearity, uncertainty, time delay and interconnection. Therefore, the study of complex interconnected systems has become increasingly important. The interest in this subject has been revived by new developments in nonlinear systems and control. This paper will review the past and recent results for both linear and nonlinear large-scale interconnected systems: decentralised schemes based on modern control approaches such as variable structure control, adaptive control and backstepping approaches, are covered; decentralised control for a class of interconnected systems with symmetric and similar structures is included; the decentralised control for singular large scale systems is reviewed as well. Some interesting/open issues will be highlighted for future discussion.

\section{Fundamental concept}

From the mathematical point of view, a nonlinear large scale interconnected system composed of $N n_{i}{ }^{\text {th }}$ order subsystems can be described by

$$
\begin{gathered}
\dot{x}_{i}=f_{i}\left(t, x_{i}\right)+g_{i}\left(t, x_{i}\right)\left(u_{i}+\Delta g_{i}\left(t, x_{i}\right)\right) \\
+\Delta f_{i}\left(t, x_{i}\right)+\sum_{\substack{j=1 \\
j \neq i}}^{N} \zeta_{i j}\left(t, x_{j}\right) \\
y_{i}=h_{i}\left(x_{i}\right), \quad i=1,2, \ldots, N
\end{gathered}
$$

where $x_{i} \in \Omega_{i} \subseteq \mathbb{R}^{n_{i}}$ ( $\Omega_{i}$ is a neighbourhood of the origin) and $u_{i} \in \mathbb{R}^{m_{i}}, y_{i} \in \mathbb{R}^{p_{i}}$ are the states, inputs and outputs of the $i^{\text {th }}$ subsystem respectively for $i=1,2, \ldots, N$. All the matrix functions $g_{i}(\cdot) \in \mathbb{R}^{n_{i} \times m_{i}}$ and the nonlinear vectors $f_{i}(\cdot) \in \mathbb{R}^{n_{i}}$ and $h_{i}(\cdot) \in \mathbb{R}^{p_{i}}$ with $h_{i}(0)=0$, are known. The terms $\Delta g_{i}(\cdot)$ and $\Delta f_{i}(\cdot)$ represent the matched and the mismatched uncertainties respectively. The term $\sum_{\substack{j=1 \\ j \neq i}}^{N} \zeta_{i j}\left(t, x_{j}\right)$ represents the interconnection of the $i^{\text {th }}$ subsystem. It is assumed that all the nonlinear functions are smooth enough such that the unforced systems have unique continuous solutions.

Firstly, the following basic definitions are introduced for the system (1).

Definition 1: Consider system (1). The system

$$
\begin{aligned}
\dot{x}_{i}= & \left.f_{i}\left(t, x_{i}\right)+g_{i}\left(t, x_{i}\right)\left(u_{i}+\Delta g_{i}\left(t, x_{i}\right)\right)\right) \\
& +\Delta f_{i}\left(t, x_{i}\right) \\
y_{i}= & h_{i}\left(x_{i}\right), \quad i=1,2, \ldots, N
\end{aligned}
$$

is called the $i^{\text {th }}$ isolated subsystem of system (1), and the system

$$
\begin{aligned}
& \dot{x}_{i}=f_{i}\left(t, x_{i}\right)+g_{i}\left(t, x_{i}\right) u_{i} \\
& y_{i}=h_{i}\left(x_{i}\right), \quad i=1,2, \ldots, N
\end{aligned}
$$

is called the $i^{\text {th }}$ nominal isolated subsystem of system (1).

It is well known that one of the main problems for interconnected systems is establishing under what conditions the interconnected system (1) exhibits the desired performance if all the isolated subsystems (2) or all the isolated nominal subsystems (3) have the performance. Therefore, how to deal with interconnection is one of the main concerned problems in decentralised control.

Definition 2: Consider system (1). If there exist controllers for each subsystems depending upon the state of the subsystems only, i.e.,

$$
u_{i}=u_{i}\left(t, x_{i}\right), \quad i=1,2, \ldots, N
$$

such that the corresponding closed-loop system formed by applying the control $u_{i}$ in (4) to system (1), has the desired performance, then the control (4) forms a decentralised static state feedback control scheme. If the controllers in (4) have the form

$$
u_{i}=u_{i}\left(t, y_{i}\right), \quad i=1,2, \ldots, N
$$

that is, each local controller depends upon the output of the subsystem only, then they are called decentralised static output feedback controllers. Furthermore, if there exist dynamical systems

$$
\dot{\hat{x}}_{i}=\phi_{i}\left(t, \hat{x}_{i}, u_{i}, y_{i}\right), \quad i=1,2, \ldots, N
$$

where controllers

$$
u_{i}=u_{i}\left(t, \hat{x}_{i}, y_{i}\right), \quad i=1,2, \ldots, N
$$

are such that the corresponding closed-loop system formed by applying the control $u_{i}$ in (7) with $\hat{x}$ given by dynamical system (6) to the system (1), has the desired performance, then the control (6) and (7) is called a decentralised dynamic output feedback control scheme. Specifically, if (6) is an observer of the system (1), then a decentralised observer-based control results. 
It is straightforward to see, according to Definition 2 above, that it is required that the dynamical systems (6) are decoupled in a decentralised dynamical output feedback scheme. It should be mentioned that in some of the existing work, see for example Yan et al. (2000) and Yan and Xie (2003), the designed dynamical systems (6) are not decoupled (in fact they are interconnected systems). In this case, the developed controller is sometimes still called a decentralised control. However, in precise terms, such a class of controllers is not decentralised because there exists information transfer between subsystems in the dynamical system design (see e.g., Yan et al., 2000; Yan and Xie, 2003).

Several decades ago, most work on decentralised control focused on linear interconnected systems due to the limitation of available control paradigms that were able to deal with nonlinearity. However, the dynamics of large scale natural and engineered interconnected systems are usually highly nonlinear. It is not only the structure of the system and interconnections which produce complexity but also the nonlinearity of the dynamics themselves. It is clear that although a linear dynamics may approximate the orbit of a nonlinear system locally, it does not permit the existence of the multiple states observed in real networks and does not accommodate global properties of the system. Such global properties can be crucial because they may become significant when the system is perturbed or a subsystem enters a failure state. Increasing requirements on system performance coupled with the ability to mode and simulate reality by means of complex, possibly nonlinear, interconnected systems models has motivated increasing contributions in the study of such systems. This interest has been further stimulated by the simultaneous development of nonlinear systems theory and the emergence of powerful mathematical and computational tools which render the formal and constructive study of nonlinear large scale systems increasingly possible (see Yan et al., 2013).

In order to explain the decentralised control concept, the following schematic diagram in which the interconnected system has three subsystems, is produced to show that in static decentralised output feedback control, the local control $u_{i}$ of the $i^{\text {th }}$ subsystem only uses local output information $y_{i}$; no output information $y_{j}(j \neq i)$ is involved in the design. From Figure 1, it is clear to see that there is no local output information transfer between the local controllers $u_{i}$ and $u_{j}(i \neq j)$ for $i, j=1,2,3$.

\section{Decentralised schemes based on modern control approach}

Many control approaches have been applied to large scale interconnected systems to form decentralised control schemes. In this section, decentralised schemes based on variable structure control, adaptive control and the backstepping approach will be briefly reviewed.

Figure 1 Decentralised static output feedback schematic diagram for interconnected systems with three subsystems (see online version for colours)

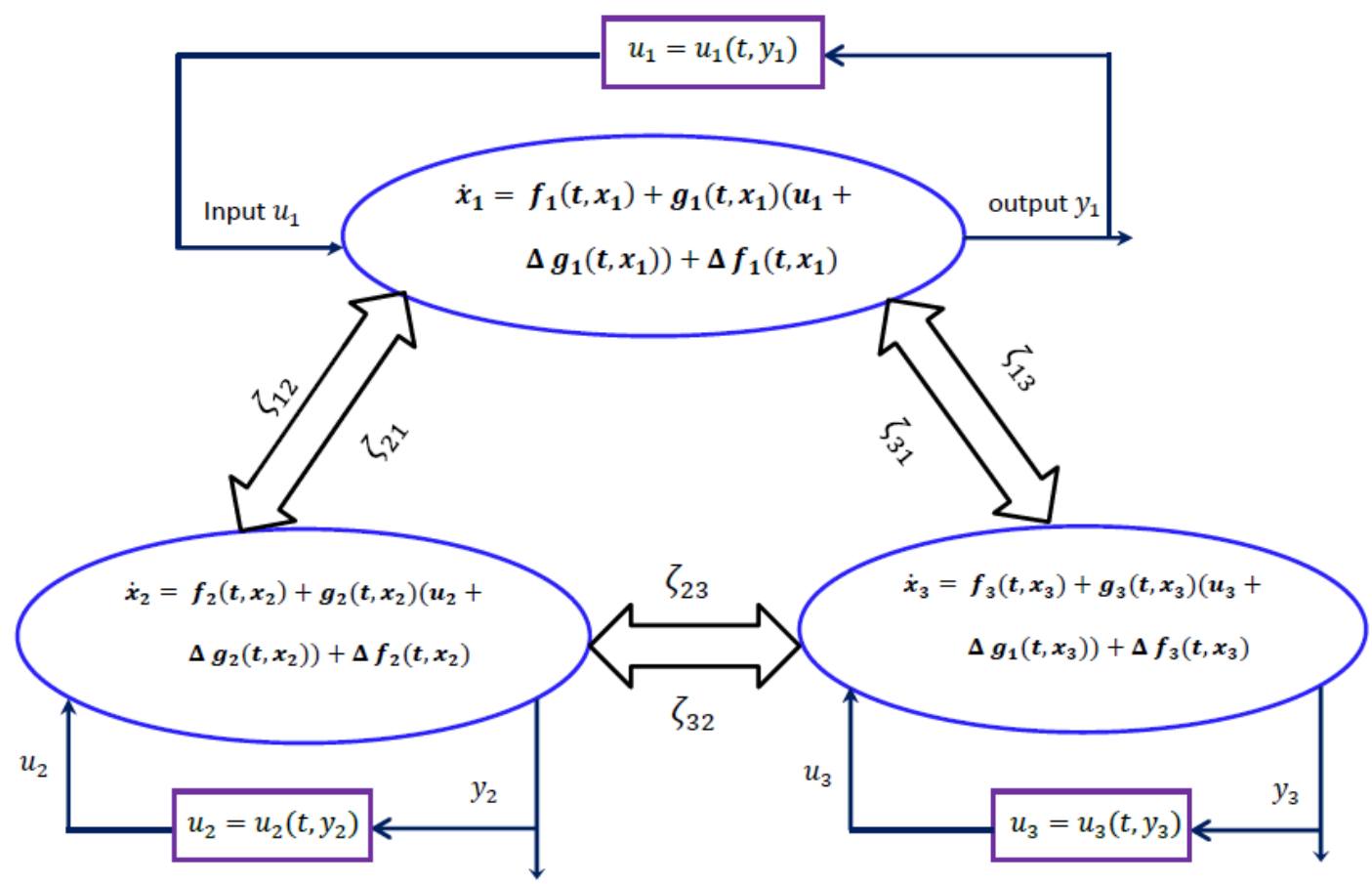




\subsection{Decentralised variable structure control}

Variable structure control has been recognised as an efficient tool in control design. The main advantages include (see Edwards et al., 2006; Edwards and Spurgeon, 1998):

- $\quad$ low sensitivity to system parameters and perturbations, eliminating the requirement for exact modelling

- the possibility of decoupling the original system into two subsystems with lower dimension

- many controllers ensure finite time convergence to the switching surface and can be straightforwardly implemented.

Sliding mode control is a particular type of variable structure control. This approach consists of two steps (Edwards and Spurgeon, 1998; Utkin, 1992; Hung et al., 1993):

1 the design of a sliding surface such that the system possesses the desired performance when it is restricted to the surface

2 the design of a variable structure control which drives the system trajectory to the sliding surface in finite time and maintains a sliding motion thereafter.

Research on decentralised control using variable structure techniques for large-scale interconnected systems can be traced back to the early 1980s (Richter et al., 1981). It should be noted that there are some decentralised variable structure controllers which may not result in sliding motion (see Hsu and Fu, 2006; Yan et al., 1998, 2013; Yan and Dai, 1998). Static output feedback controllers are proposed in Yan et al. (1998, 2013) and Yan and Dai (1998) to stabilise interconnected systems where the focus is on applying the nonlinear bounds on the uncertainty and interconnections to enhance the robustness. Specifically both the isolated subsystems and interconnections are fully nonlinear in Yan and Dai (1998) and Yan et al. (2013). A tracking problem is considered in Hsu and $\mathrm{Fu}$ (2006) where decentralised state feedback controller are designed for robot manipulators.

Broadly, the literature can be separated into state feedback (Lee and Wang, 1993; Lee, 1995; Hsu, 1997; Akar and Ozguner, 2002; Matthews and Decarlo, 1988), static output feedback (Yan et al., 2012, 2004) and dynamical/observer-based output feedback (Lee, 1995; Yan et al., 2006). Since all the system state variables are available in the state feedback control design, the requirement of state feedback control for the considered system is usually relaxed and the developed results have higher robustness when compared with that of output feedback control. It is well known that the fundamental question of the existence of a static output feedback controller for a given triple $(A, B, C)$ is still open - even in the single-input single-output case (Syrmos et al., 1997) although the standard constrained Lyapunov problem has been well discussed in Galimidi and Barmish (1986) and Edwards et al. (2007). Therefore, most of the decentralised output feedback schemes focus on dealing with various uncertainties and interconnections instead of the control design for the nominal isolated subsystem. Approaches consider under what conditions the interconnected system has the desired performance if the isolated nominal subsystems have the required performance (see Yan et al., 2004, 2006). A decentralised sliding-mode-based output-feedback control scheme for discrete time systems is available in Mahmoud and Qureshi (2012) which is built on the work in Yan et al. (2009). As for the dynamical/observer-based feedback control, the results developed usually have higher robustness and lower conservatism when compared with static output feedback because for dynamical output feedback, not only the output information can be employed in the design but the dynamical information can also be employed.

It is clear that a number of decentralised variable structure strategies have been obtained. The global reachability condition for interconnected systems was first proposed in $\mathrm{Xu}$ et al. (1990). Later, it was modified as follows

$$
\sum_{i=1}^{N} \frac{s_{i}^{T}\left(x_{i}\right) s_{i}\left(x_{i}\right)}{\left\|s_{i}(x)\right\|}<0
$$

in Lee and Wang (1993) and the simple proof was given in Hsu (1997), where $s_{i}\left(x_{i}\right)$ can be considered as a decentralised sliding function which is usually designed based on the centralised sliding surface design approaches given in Edwards and Spurgeon (1998) and Zak and Hui (1993). The reachability condition (8) has been extensively employed in decentralised sliding mode control design to derive the reachability condition but it may not guarantee that the sliding surface is reached in finite time. In order to get a decentralised control strategies, the decentralised (or local) sliding surface $s_{i}\left(x_{i}\right)=0$ is designed based on the nominal system of the $i^{\text {th }}$ subsystems in nearly all the existing work. Then the composite sliding function is given by

$$
S=\left(s_{1}^{T}\left(x_{1}\right), s_{2}^{T}\left(x_{2}\right), \ldots, s_{n}^{T}\left(x_{n}\right)\right)^{T}
$$

which can be considered as the decentralised sliding function. Whether it is possible to design a sliding surface in a general form as

$$
s\left(x_{1}, x_{2}, \cdots, x_{n}\right)=0
$$

to implement a decentralised control scheme, is still open even for a large scale interconnected system with special structure. Moreover, when global results are sought, strong conditions are required on the nominal subsystems to satisfy the reachability condition globally when a static output feedback controller is designed, as presented in Yan et al. (2009, 2012) (see Assumption 4 in Yan et al., 2012). This condition was later employed in Mahmoud and Qureshi (2012) for a class of discrete time interconnected systems. How to relax this condition is still an open problem. 
Finally, it should be pointed out that higher order sliding mode control has been well studied (see, e.g., Levant and Fridman, 2010; Bartolini et al., 2003; Dinuzzo and Ferrara, 2009). In addition, many results concerning decentralised schemes for interconnected systems have been obtained as mentioned above (Yan and Dai, 1998; Yan et al., 1998; Mahmoud, 2009), even for fully nonlinear interconnected systems (Yan et al., 2010). However, the results of applying decentralised higher order sliding mode control to large scale interconnected systems are very limited. Specifically, the results of decentralised control for nonlinear time delay interconnected systems using higher order sliding mode techniques are still not available. Study on this area is at its initial stage and thus could be interesting and full of challenge from both viewpoints of theory and application.

\subsection{Decentralised adaptive control}

Adaptive control was conceived in the 1950s. It has been most successful for plant models in which unknown/uncertain parameters appear linearly (Kristic et al., 1995). Unlike robust control, adaptive control (Liu and Lin, 2013) does not require priori information and knowledge for the bounds on these uncertain/unknown parameters. It is well-known that robust control guarantees that the system has desired performance if the uncertainties change within given bounds. In this case, the control law does not need to change. However, adaptive control is concerned with a control law that changes itself. The foundation of adaptive control is parameter estimation. The main idea of adaptive control is to maintain a desired system performance in the presence of uncertainties or unknown variation in plant parameters. When the parameters change, the system adapts to the changes in the system to maintain performance. Effectively, there is adaptation in the controller setting subject to the performance of the closed-loop system. How the controller parameters change is determined by the adaptive laws which are often designed based on the requirements for the designed adaptive control system (Ding, 2013).

When unknown parameters appear in large scale interconnected systems and the bounds on the uncertainties are not available, decentralised adaptive control is a good choice to maintain the system performance. A decentralised adaptive robust control scheme is proposed for a class of large-scale interconnected systems to guarantee the corresponding closed-loop systems ultimate boundedness in Wu (2002), Hua et al. (2005) and Liu and Xie (2010). Interconnected systems with time delay are considered in Wu (2002) and Hua et al. (2005) where it is required that the interconnections satisfy the matching condition. A decentralised adaptive output feedback controller to solve the asymptotic tracking problem was proposed in Jiang (2000) where the matching condition is not required. Decentralised model reference adaptive control schemes are proposed in Wen and Soh (1999) and Chou and Cheng (2003) which do not need information on the upper bound of the perturbations. The robust tracking problem is solved using model reference adaptive variable structure control but matching conditions are required for the local reference model in Chou and Cheng (2003). Shi and Singh (1992) proposed a decentralised adaptive controller to deal with a class of interconnected systems which are bounded by $p^{\text {th }}$ order polynomials in the states. Global stabilisation is considered in Wen and Soh (1999) where there is no restriction on the relative degree of the nominal subsystem. In this paper, parameter projection together with static normalisation is employed.

In recent years, adaptive techniques have been successfully combined with intelligent control techniques such as fuzzy control (Liu and Lin, 2013), neural networks (Chai and Qiao, 2013) and learning control (Yan et al., 2001) to design decentralised controllers. Great attention has been paid to this area and many results have been achieved, for example, Tong et al. (2010), Zhou et al. (2012), Mehraeen et al. (2011) and Liu et al. (2014). It is possible that correct limitations on the interconnections in decentralised adaptive control strategies may be overcome by the use of intelligent control concepts.

\subsection{Backstepping approach-based decentralised control}

Backstepping is a technique developed by Petar V. Kokotovic and others at the beginning of 1990s (Kokotovic, 1992; Lozano and Brogliato, 1992). Compared with variable structure techniques and adaptive control, the history of the backstepping approach is relatively short. This approach requires that the considered system has a special structure called strict-feedback form described as follows

$$
\begin{aligned}
\dot{x} & =f_{0}(x)+g_{0}(x) z_{1} \\
\dot{z}_{1} & =f_{1}\left(x, z_{1}\right)+g_{1}\left(x, z_{1}\right) z_{2} \\
\dot{z}_{2} & =f_{2}\left(x, z_{1}, z_{2}\right)+g_{2}\left(x, z_{1}, z_{2}\right) z_{3} \\
& \vdots \\
\dot{z}_{k-1} & =f_{k-1}\left(x, z_{1}, \cdots, z_{k-1}\right)+g_{k-1}\left(x, z_{1}, \cdots, z_{k-1}\right) z_{k} \\
\dot{z}_{k} & =f_{k}\left(x, z_{1}, \cdots, z_{k}\right)+g_{k}\left(x, z_{1}, \cdots, z_{k}\right) u
\end{aligned}
$$

where $x \in \mathbb{R}^{n}$ is vector and $z_{i}$ are scalars for $i=1,2, \ldots, k$, and the variables $x$ and $z_{i}$ with $i=1,2, \ldots, k$ form the system state and $u \in \mathbb{R}$ is system input. The functions $f_{i}(\cdot)$ vanish at the origin and $g_{i}(\cdot)$ are non-zero over the domain of interest for $i=0,1,2, \ldots, k$.

It should be noted that a backstepping controller can not be explicitly expressed in terms of equations because the controller is developed step by step using recursive design. This may limit its application. However, backstepping design methods can provide a systematic procedure to design stabilising controllers, following a step by step recursive algorithm, whereby the feedback control laws and Lyapunov functions can be constructed systematically. Moreover, this approach can be employed to relax the matching condition when compared with the traditional Lyapunov design approach. A major advantage of backstepping is that it has the flexibility 
to avoid cancellation of useful nonlinearities and achieve desirable regulation and tracking properties (Zhou and Wen, 2008a).

Backstepping techniques require the considered system to have a special structure and thus most work considers parametric uncertainty instead of unstructured uncertainty. It is well known that for uncertain/unknown parameters, adaptive methods are much powerful as pointed out in the last section. Therefore large scale interconnected systems considered in this area usually have two characteristics:

1 all the isolated nominal subsystems have or can be transformed to the strict feedback form

2 the considered systems involve parametric uncertainty.

These two features require adaptive updated laws to estimate the uncertain parameters and thus the approaches usually involve both adaptive and backstepping techniques (Liu and Xie, 2010; Wen et al., 2009; Zhou and Wen, 2008b; Wang and Soh, 1997; Zhou, 2008; Ye, 1999). A decentralised PID controller is proposed using backstepping techniques in Zhang et al. (2007) where the considered system model is expressed in the frequency domain. Recently, some authors have combined backstepping techniques with intelligent control such as fuzzy control and neural network to deal with interconnected systems (Mehraeen et al., 2011; Tong et al., 2011; Zhou et al., 2012; Tong et al., 2010).

As one of the advantages of backstepping approach is that it has the flexibility to achieve regulation and tracking properties, the relevant decentralised strategies have also received much attention. The first decentralised control based on backstepping without any requirement on subsystem relative degree was reported in Wen (1994) where perfect regulation is obtained. Global decentralised output regulation is considered in Ye (1999) where the reference input and disturbances are the sum of finitely sinusoidal functions with unknown amplitudes and frequencies. Observer-based decentralised disturbance attenuating output-feedback tracking controllers are designed in Jiang (2002); Krishnamurthy and Khorrami (2003). By combining a neural network approach (Rekik et al., 2007), decentralised backstepping controllers are proposed in Tong et al. (2011) and Chen and Li (2008) where the tracking error only converges to a bounded region of the origin. The result developed in Zhou and Wen (2008b) expressed the tracking error as a function of design parameters which makes it possible to adjust the tracking transient performance.

Compared with decentralised variable structure control and decentralised adaptive control, the domain of backstepping-based decentralised control is more immature and the obtained results are fewer.

\section{System structure-based decentralised control}

System structure plays an important role in control design. One of the fundamental properties of large scale interconnected systems in the real world is that the operational characteristics of the components (subsystems) may be very similar and indeed symmetrically identical. For example, in a manufacturing process, in order to produce the same engineering components in large quantities, many identical or similar machine tools (isolated subsystems) are interconnected together to form a large-scale system to complete the task. The key structural characteristics existing in large-scale interconnected systems relate to symmetry (Lunze, 1992) and similarity (Yan et al., 2000). By using such structural characteristics, design and analysis for complex interconnected systems can be simplified.

Study on symmetric interconnected systems dates back to at least 1980 (Baliga and Rao, 1980). Based on the circulant structure of transfer function matrix, a class of symmetrically interconnected plants is introduced in Hovd and Skogestad (1994). This study shows that the symmetric structure employed produces a dramatic reduction in dimension for interconnected systems composed of a set of subsystems. It should be pointed out that the study of symmetric interconnected systems has mainly focused on the linear case and a suitable formulation for the symmetric structure of nonlinear interconnected systems has not been available. Symmetric interconnected systems can be described by

$$
\begin{aligned}
& \dot{x}_{i}=A_{1} x_{i}+B_{1} u_{i}+\sum_{\substack{j=1 \\
j \neq i}}^{N} H x_{j} \\
& y_{i}=C_{1} x_{i}, \quad i=1,2, \ldots, N
\end{aligned}
$$

where $x_{i} \in \mathbb{R}^{n}, u_{i} \in \mathbb{R}^{m}$ and $y_{i} \in \mathbb{R}^{p}$ are the system states, inputs and outputs respectively, and $A_{1}, B_{1}, C_{1}$ and $H$ are constant matrices with appropriate dimensions. System (9) can be written in a compact form as

$$
\begin{aligned}
& \dot{x}=A x+B u \\
& y=C x
\end{aligned}
$$

where $x=\operatorname{col}\left(x_{1}, x_{2}, \cdots, x_{N}\right), u=\left(u_{1}^{T}, u_{2}^{T}, \cdots, u_{N}^{T}\right)^{T}$, $y=\left(y_{1}^{T}, y_{2}^{T}, \cdots, y_{N}^{T}\right)^{T}$ and

$$
\begin{gathered}
A=\left[\begin{array}{ccccc}
A_{1} & H & H & \cdots & H \\
H & A_{1} & H & \cdots & H \\
H & H & A_{1} & \cdots & H \\
\vdots & \vdots & \vdots & \ddots & \vdots \\
H & H & H & \cdots & A_{1}
\end{array}\right] \\
B=\operatorname{diag} \underbrace{\left\{B_{1}, B_{1}, \cdots, B_{1}\right\}}_{N}
\end{gathered}
$$

$$
C=\operatorname{diag} \underbrace{\left\{C_{1}, C_{1}, \cdots, C_{1}\right\}}_{N}
$$


This class of large-scale interconnected systems is representative of systems which widely exist in the real world (Sundareshan and Elbanna, 1991) such as the multi-machine power system discussed in Mohadjer and Johnson (1983) and industrial robot manipulators considered in Vukabratovic and Stokic (1982). The formulation has thus been extensively studied, and many results have been achieved, for example, Liu (1992), Yang and Zhang (1995) and Huang et al. (1999). The output regulation problem was solved for a class of strongly coupled symmetric interconnected systems in Liu (1992). Yang and Zhang (1995) proposed a set of conditions described by two Riccati equations under which a control scheme is developed such that the corresponding closed-loop system is asymptotically stable. By employing the symmetric structure of the interconnected systems, a set of sufficient conditions for the existence of a decentralised $H_{\infty}$ controller is derived, and the problem of fault tolerance of the resulting system was considered in Huang et al. (1999). Recently, pole assignment for uncertain symmetric circulant composite systems is considered in Liu et al. (2006) where a decentralised stabilisation controllers are designed to place all of the poles of the system into a specified disk which is also called $D$ stabilisation.

A class of interconnected systems with similar subsystems were studied in Yan et al. (1997, 1998). The exact definition of similar structure for nonlinear interconnected systems was introduced for the first time in Yan (1996), and can be briefly summarised as follows.

Consider the following two control systems

$$
\sum_{1}:\left\{\begin{array}{l}
\dot{x}_{1}=f_{1}\left(t, x_{1}\right)+g_{1}\left(t, x_{1}\right) u_{1} \\
y_{1}=h_{1}\left(x_{1}\right)
\end{array}\right.
$$

and

$$
\sum_{2}:\left\{\begin{array}{l}
\dot{x}_{2}=f_{2}\left(t, x_{2}\right)+g_{2}\left(t, x_{2}\right) u_{2} \\
y_{2}=h_{2}\left(x_{2}\right)
\end{array}\right.
$$

where $x_{1}, x_{2} \in \mathbb{R}^{n}, u_{1}, u_{2} \in \mathbb{R}^{m}$ and $y_{1}, y_{2} \in \mathbb{R}^{p}$ are the system states, inputs and outputs respectively.

Definition 3: Systems $\sum_{1}$ and $\sum_{2}$ are said to have similar structure, if there exists a diffeomorphism $T: x_{2} \mapsto x_{1}$, a $C^{\infty}$ function vector $\alpha(\cdot) \in \mathbb{R}^{m}$ and a $C^{\infty}$ non-singular function matrix $\beta(\cdot) \in \mathbb{R}^{m \times m}$ such that the closed-loop system formed by applying the control

$$
u_{1}=\alpha\left(x_{1}\right)+\beta\left(x_{1}\right) u_{2}
$$

to system $\sum_{1}$, can be expressed in the form of $\sum_{2}$ in the coordinate $x_{2}$ defined by $T$.

Definition 4: Systems (1) are called similar interconnected systems or interconnected systems with similar structure if all their nominal subsystems have similar structure.

For large-scale interconnected/composite systems with similar structure, two types of discontinuous nonlinear decentralised controller with holographic structure are proposed to stabilise the system asymptotically by exploiting the similar structure (Yan et al., 1999; Wang and Zhang, 2000). These are easily implemented compared with general nonlinear controllers under the assumption that all the system states are available for design (Yan et al., 1999; Wang and Zhang, 2000). A static output feedback controller with holographic structures was proposed in Yan et al. (1998) and dynamical output feedback control schemes have also been presented in Yan and Xie (2003) and Yan et al. (2000). However, the similar structure defined through Definitions 3 and 4 does not consider the structure of the interconnections. It may be very interesting to study how the structure of interconnections affects the decentralised control design and the interconnected system analysis. Modelling the similar structure of nonlinear interconnections is challenging but may be useful for the study of decentralised control.

It should be noted that for a symmetrically interconnected system (9), if the matrix $A$ in system (10) has the following form,

$$
A=\left[\begin{array}{ccccc}
A_{o} & L & L & \cdots & L \\
M & A_{1} & H & \cdots & H \\
M & H & A_{1} & \cdots & H \\
\vdots & \vdots & \vdots & \ddots & \vdots \\
M & H & H & \cdots & A_{1}
\end{array}\right]
$$

where $A_{0}, M$ and $L$ are constant matrices with appropriate dimensions, then the corresponding systems are also called similar interconnected systems (Lunze, 1989). Study of such a class of systems has obtained interesting results (see e.g., Lunze, 1989; Yang and Zhang, 1995, 1996) and a class of interconnected systems with similar nodes is considered in Wang et al. (2012). It should be noted that in recent years the study of symmetric or similar interconnected systems has been limited. The means to describe a similar interconnected system by considering the structure of the interconnections is still an open problem.

\section{Decentralised control of singular systems}

A linear singular control system with $N$ channels can be described by

$$
\begin{aligned}
& E \frac{d x(t)}{d t}=A x(t)+\sum_{i=1}^{N} B_{i} u_{i}(t) \\
& y_{i}(t)=C_{i} x(t), \quad i=1,2, \ldots, N
\end{aligned}
$$

where $x(t) \in \mathbb{R}^{n}$ denotes the system state; $u_{i}(t) \in \mathbb{R}^{m_{i}}$ and $y_{i}(t) \in \mathbb{R}^{l_{i}}$ are the input and output of the $i^{\text {th }}$ channel, respectively, for $i=1,2, \cdots, N$. The matrices $E$, $A, B_{i}$ and $C_{i}$ are constant with appropriate dimensions. Specifically, the matrix $E$ is singular and $0<\operatorname{rank}(E)<n$. It is assumed that the system (11) is regular, i.e., $\operatorname{det}(s E-$ $A) \neq 0$. Then, the existence and uniqueness of the solution to system (11) is guaranteed and this solution may take the form of an exponent solution, impulsive solution and/or static solution. 
System (11) has been studied extensively where most of the research work focuses on decentralised output feedback control design, which is to design a control

$$
u_{i}=K_{i} y_{i}
$$

where $K_{i}$ for $i=1,2, \ldots, N$ are design parameters, such that the corresponding closed-loop system has the desired performance (Guan et al., 1995; Zhang, 1997). Compensator-based decentralised control was studied for singular interconnected systems in Gao et al. (1997).

Such linear singular control systems (11) were first represented and studied by Chang and Davison (1988), where the concepts of generalised decentralised finite and impulsive fixed modes were proposed to derive conditions under which the systems can be stabilised by decentralised compensator-based feedback control. The decentralised robust servomechanism problem was solved simultaneously. Generalised decentralised finite fixed modes were further characterised in Zhang (1990), and the concepts of decentralised impulsive controllability and observability were presented and studied in Chang and Davison (1989) where it was shown that if the considered system is complete, then the system does not have decentralised fixed modes if and only if it is decentralised controllable and observable. Based on the work in Zhang (1990) and Chang and Davison (1989), the decentralised quadratic stabilisation problem for a class of interconnected singular systems with uncertainty is investigated in Yasuda and Noso (1996) in which it was shown that quadratic stability requires that the considered system has neither decentralised unstable finite fixed modes nor decentralised impulsive fixed modes. The internal properness and stability of system (11) were discussed, and a set of necessary and sufficient conditions was presented in Gao et al. (1997) such that the system can be stabilised by decentralised compensator-based feedback control. Decentralised stabilisation of singular and time-delay large-scale systems with impulsive solutions was discussed in Guan et al. (1995) where the designed controllers are independent of time delay. Regularisation and impulse eliminating problems are considered using decentralised controllers in $\mathrm{Yu}$ (2005) and $\mathrm{Yu}$ and Wang (2005) respectively. Concerning decentralised $\mathrm{H}_{2}$ and $H_{\infty}$ control for singular systems, some recent work is available in Zhai et al. (2006) and Chen et al. (2009) and the references therein. As for the discrete time case with parametric uncertainties, robust stabilising controllers were designed in Wo et al. (2007). Recently, a decentralised observer for the system (11) was considered in Mansouri et al. (2013) using LMI techniques, and a fuzzy decentralised control scheme was proposed for a class of nonlinear singular interconnected systems in Wang and Zhang (2001). It has been found in Labibiy et al. (2003) that a singular system representation can be used as a suitable tool to solve some control problems such as $H_{\infty}$ control problem. Some earlier work for singular systems (11) was summarised in Zhang (1997).

\section{Applications}

Decentralised control is powerful in dealing with large scale interconnected systems and thus has been widely used in various application areas. As a typical interconnected system, multi-machine power systems have received much attention and many decentralised control strategies have been developed including state feedback, static output feedback and dynamical output feedback control approaches (Wang et al., 1997; Yan et al., 2004; Jiang and Wu, 2004; Liu et al., 2012). A multi-link robot can be modelled as an interconnected system, and various decentralised control schemes have been developed to study the control of robot manipulators (Hsu and $\mathrm{Fu}, 2006$; Yang et al., 2012). Decentralised $\mathrm{H}_{2} / \mathrm{H}_{\infty}$ tracking control has been applied to car-like mobile robots in Hwang and Chang (2007) and a decentralised cooperative control scheme has been developed for a team of mobile robots in Rezaee and Abdollahi (2014). A variable structure adaptive fuzzy logic control approach for designing a decentralised controller for load frequency control of interconnected power areas in El-Metwally (2010) to improve the dynamic performance of the frequency and tie-line power flow under a sudden load change in the power areas. In addition, decentralised control has been applied to other practical systems such as a coupled inverted pendulum system (Yan et al., 2003; Yoo et al., 2009; Li et al., 2011), wind turbine (Wu et al., 2008), vibration isolation system (Kim et al., 2001), a platooning vehicles system (Yan and Edwards, 2008; Ghasemi et al., 2013), river pollution control (Mahmoud and Hassen, 1986), chemical process control (Hudon and Bao, 2012; Zhang et al., 2013; Lunze, 1992), satellite attitude control (Wu et al., 2012) and flight control (Vukobratovic and Stojic, 1986; Boskovic and Mehra, 2010) to name a few. In recent years, decentralised control strategies have been employed in bio-engineering systems (Sato et al., 2012; Nekoukar and Erfanian, 2012). Widening application of decentralised control will stimulate new developments in the area.

\section{Conclusions}

In this paper, the past and recent results from the domain of decentralised control have been reviewed. The focus has been upon the modern control approaches and system structure. It is clear that the case of decentralised control continues to attract increasing attention. This paper cannot cover all areas owing to space limitations, and areas not covered include decentralised control of discrete event systems, decentralised multi-agent systems, decentralised observation and decentralised supervisory control.

It should be pointed out that decentralised control has been studied for many years and is an established area. Many centralised results have been extended to the decentralised case to tackle important problems relevant to large scale interconnected systems. However, from the theoretical point of view, novel and innovative approaches for comparing alternative decentralised information and 
decision structures are required. Novel mathematical tools may be necessary for dealing with the interconnections, when decentralised strategies are considered. If a significant step-change in progress is to be achieved in the study of control of large scale systems, it is perhaps the case that the somewhat incremented step of applying existing centralised paradigms to decentralised problems has limited the results that can be achieved and that a new decentralised paradigm is required. This will be the topic of future research.

\section{Acknowledgements}

This work is supported by the EPSRC via grant reference $\mathrm{EP} / \mathrm{J} 017477 / 1$ and the Royal Academy of Engineering of UK via grant reference 12/13RECI027.

\section{References}

Akar, M. and Ozguner, U. (2002) 'Decentralized sliding mode control design using overlapping decomposition', Automatica, Vol. 38, No. 10, pp.1713-1718.

Bakule, L. (2008) 'Decentralized control: an overview', Annual Reviews in Control, Vol. 32, No. 1, pp.87-98.

Baliga, G. and Rao, M. (1980) 'On symmetric and unity interconnections between three nonlinear subsystems', Automatica, Vol. 16, No. 6, pp.711-713.

Bartolini, G., Pisano, A., Punta, E. and Usai, E. (2003) 'A survey of applications of second-order sliding mode control to mechanical systems', Int. J. Control, Vol. 76, Nos. 9-10, pp.875-892.

Boskovic, J. and Mehra, R. (2010) 'A decentralized fault-tolerant control system for accommodation of failures in higher-order flight control actuators', IEEE Trans. on Control Systems Technology, Vol. 18, No. 5, pp.1103-1115.

Chai, W. and Qiao, J. (2013) 'Non-linear system identification and fault detection method using rbf neural networks with set membership estimation', Int. J. Modelling, Identification and Control, Vol. 20, No. 2, pp.114-120.

Chang, T. and Davison, E.J. (1988) 'Decentralized control for descriptor type systems', in Proceedings of 25th IEEE Conference on Decision and Control, pp.1176-1181, Athens, Greece.

Chang, T. and Davison, E.J. (1989) 'Algebraic characterizations of fixed modes in decentralized descriptor systems', in Proceedings of 28th IEEE Conference on Decision and Control, pp.866-871, Florida, USA.

Chen, N., Gui, W.H. and Zhai, G.S. (2009) 'Robust decentralized $\mathrm{H}_{\infty}$ control for interconnected descriptor systems with norm-bounded uncertainties', Asian Journal of Control, Vol. 11, No. 1, pp.78-88.

Chen, W. and Li, J. (2008) 'Decentralized output-feedback neural control for systems with unknown interconnections', IEEE Trans. on Systems, Man and Cybernetics, Vol. 38, No. 1, pp.258-266.

Chou, C.H. and Cheng, C.C. (2003) 'A decentralized model reference adaptive variable structure controller for large-scale time-varying delay systems', IEEE Trans. on Automat. Control, Vol. 48, No. 7, pp.1213-1217.
Ding, Z. (2013) Nonlinear and Adaptive Control Systems, The Institution of Engineering and Technology (IET Control Engineering Series 84), London.

Dinuzzo, F. and Ferrara, A. (2009) 'Higher order sliding mode controllers with optimal reaching', IEEE Trans. on Automat. Control, Vol. 54, No. 9, pp.2126-2136.

Edwards, C., Colet, E. and Fridman, L. (2006) Advances in Variable Structure and Sliding Mode Control, Lecture Notes in Control and Information Sciences, Vol. 334, Springer-Verlag, Berlin Heidelberg.

Edwards, C. and Spurgeon, S.K. (1998) Sliding Mode Control: Theory and Applications, Taylor and Francis Ltd., London.

Edwards, C., Yan, X.G. and Spurgeon, S.K. (2007) 'On the solvability of the constrained Lyapunov problem', IEEE Trans. on Automat. Control, Vol. 52, No. 10, pp.1982-87.

El-Metwally, K. (2010) 'A variable structure adaptive fuzzy logic stabiliser for a two-area load frequency control problem', Int. J. Modelling, Identification and Control, Vol. 9, No. 2, pp.168-175.

Galimidi, A.R. and Barmish, B.R. (1986) 'The constrained Lyapunov problem and its application to robust output feedback stabilization', IEEE Trans. on Automat. Control, Vol. 31, No. 5, pp.410-419.

Gao, Z.W., Wang, X.L., Wang, J. and Li, G.Q. (1997) 'Internal properness and stability in singular decentralized control systems', in Proceedings of the American Control Conference, pp.2520-2521, New Mexico, USA.

Garelli, F., Mantz, R. and De Battista, H. (2006) 'Limiting interactions in decentralized control of MIMO systems', $J$. of Process Control, Vol. 16, No. 5, pp.473-483.

Ghasemi, A., Kazemi, R. and Azadi, S. (2013) 'Stable decentralized control of a platoon of vehicles with heterogeneous information feedback', IEEE Trans. on Vehicular Technology, Vol. 62, No. 9, pp.4299-4308.

Guan, Z.H., Liu, Y.Q. and Wen, X.C. (1995) 'Decentralized stabilization of singular and time-delay large-scale control systems with impulsive solutions', IEEE Trans. on Automat. Control, Vol. 40, No. 8, pp.1437-1440.

Hovd, M. and Skogestad, S. (1994) 'Control of symmetrically interconnected plants', Automatica, Vol. 30, No. 6, pp.957-973.

Hsu, K.C. (1997) 'Decentralized variable-structure control design for uncertain large-scale systems with series nonlinearities', Int. J. Control, Vol. 68, No. 6, pp.1231-1240.

Hsu, S-H. and Fu, L-C. (2006) 'A fully adaptive decentralized control of robot manipulators', Automatica, Vol. 42, No. 10, pp.1761-1767.

Hua, C., Guan, X. and Shi, P. (2005) 'Robust decentralized adaptive control for interconnected systems with time delays', ASME Journal Dynamics Systems, Measurement and Control, Vol. 127, No. 4, pp.656-662.

Huang, S., Lam, J., Yang, G. and Zhang, S. (1999) 'Fault tolerant decentralized $\mathrm{H}^{\infty}$ control for symmetric composite systems', IEEE Trans. on Automat. Control, Vol. 44, No. 11, pp.2108-2114.

Hudon, N. and Bao, J. (2012) 'Dissipativity-based decentralized control of interconnected nonlinear chemical processes', Computer and Chemical Engineering, Vol. 45, pp.84-101.

Hung, J.Y., Gao, W. and Hung, J.C. (1993) 'Variable structure control: a survey', IEEE Trans. on Industrial Electronics, Vol. 40, No. 1, pp.2-22. 
Hwang, C. and Chang, L. (2007) 'Trajectory tracking and obstacle avoidance of car-like mobile robots in an intelligent space using mixed $\mathrm{H}_{2} / \mathrm{H}_{\infty}$ decentralized control', IEEE/ASME Transactions on Mechatronics, Vol. 12, No. 3, pp.345-352.

Jiang, L. and Wu, Q.H. amd Wen, J. (2004) 'Decentralized nonlinear adaptive control for multimachine power systems via high-gain perturbation observer', IEEE Trans. on Circuits and Systems (I), Vol. 51, No. 10, pp.2052-2059.

Jiang, Z.P. (2000) 'Decentralized and adaptive nonlinear tracking of large-scale systems via output feedback', IEEE Trans. on Automat. Control, Vol. 45, No. 11, pp.2122-2128.

Jiang, Z.P. (2002) 'Decentralized disturbance attenuating output-feedback trackers for large-scale nonlinear systems', Automatica, Vol. 38, No. 8, pp.1407-1415.

Kim, S., Elliott, S. and Brennan, M. (2001) 'Decentralized control for multichannel active vibration isolation', IEEE Trans. on Control Systems Technology, Vol. 9, No. 1, pp.93-100.

Kokotovic, P. (1992) 'The joy of feedback: nonlinear and adaptive', IEEE Control Systems Magazine, Vol. 12, No. 3, pp.7-17.

Krishnamurthy, P. and Khorrami, F. (2003) 'Decentralized control and disturbance attenuation for large-scale nonlinear systems in generalized output-feedback canonical form', Automatica, Vol. 39, No. 11, pp.1923-1933.

Kristic, M., Kanellakopoulos, I. and Kokotovic, P. (1995) Nonlinear and Adaptive Control Design, John Wiley and Sons, Inc., New York.

Labibiy, B., Lohmannz, B., Sedighy, K. and Maralani, P. (2003) 'Robust decentralized control of large-scale systems via $\mathrm{H}_{\infty}$ theory and using descriptor system representation', Int. J. Systems Sci., Vol. 34, No. 12, pp.705-715.

Lee, J.L. (1995) 'On the decentralized stabilization of interconnected variable structure systems using output feedback', Journal of the Franklin Institute, Vol. 332, No. 5, pp.595-605.

Lee, J-L. and Wang, W-J. (1993) 'Robust decentralized stabilization via sliding mode control', Control Theory Adv. Tech., Vol. 9, No. 3, pp.721-731.

Levant, A. and Fridman, L. (2010) 'Accuracy of homogeneous sliding modes in the presence of fast actuators', IEEE Trans. on Automat. Control, Vol. 55, No. 3, pp.810-14.

$\mathrm{Li}, \mathrm{T}$., Li, W. and $\mathrm{Bu}, \mathrm{R}$. (2011) 'A novel decentralised adaptive NN tracking control for double inverted pendulums', Int. J. Modelling, Identification and Control, Vol. 13, No. 4, pp.269-277.

Liu, D., Wang, D. and Li, H. (2014) 'Decentralized stabilization for a class of continuous-time nonlinear interconnected systems using online learning optimal control approach', IEEE Trans. on Neural Networks and Learning Systems, Vol. 25, No. 2, pp.418-428.

Liu, H., Hu, Z. and Song, Y. (2012) 'Lyapunov-based decentralized excitation control for global asymptotic stability and voltage regulation of multi-machine power systems', IEEE Trans. on Power Systems, Vol. 27, No. 4, pp.2262-2270.

Liu, L. and Xie, X. (2010) 'Decentralized adaptive stabilization for interconnected systems with dynamic input-output and nonlinear interactions', Automatica, Vol. 46, No. 6, pp.1060-1067.
Liu, M., Jing, Y. and Zhang, S. (2006) 'Pole assignment for uncertain symmetric circulant composite systems in a specified disk', IEE Proc. Part D: Control Theory Appl., Vol. 153, No. 3, pp.357-363.

Liu, S., Zhang, J.F. and Jiang, Z. (2007) 'Decentralized adaptive output-feedback stabilization for large-scale stochastic nonlinear systems', Automatica, Vol. 43, No. 2, pp.238-251.

Liu, S. and Lin, S. (2013) 'Robust adaptive controller design for uncertain fuzzy systems using linear matrix inequality approach', Int. J. Modelling, Identification and Control, Vol. 18, No. 2, pp.110-118.

Liu, X. (1992) 'Output regulation of strongly coupled symmetric composite systems', Automatica, Vol. 28, No. 5, pp.1037-1041.

Lozano, R. and Brogliato, B. (1992) 'Adaptive control of robot manipulators with flexible joints', IEEE Trans. on Automat. Control, Vol. 37, No. 2, pp.174-181.

Lunze, J. (1989) 'Stability analysis of large-scale systems composed of strongly coupled similar subsystems', Automatica, Vol. 25, No. 4, pp.561-570.

Lunze, J. (1992) Feedback Control of Large Scale Systems, Prentice Hall International (UK) Ltd., Hemel Hempstead.

Mahmoud, M. and Hassen, M. (1986) 'Decentralized water-quality control scheme', IEEE Trans. on Systems, Man and Cybernetics, Vol. 16, No. 5, pp.694-702.

Mahmoud, M.S. (2009) 'Decentralized stabilization of interconnected systems with time-varying delays', IEEE Trans. on Automat. Control, Vol. 54, No. 11, pp.2663-68.

Mahmoud, M.S. (2011) Decentralized Systems with Design Constraints, Springer-Verlag London Limited.

Mahmoud, M.S. and Qureshi, A. (2012) 'Decentralized sliding-mode output-feedback control of interconnected discrete-delay systems', Automatica, Vol. 48, No. 5, pp.808-814.

Mansouri, M., Boutat-Baddas, L., Darouach, M. and Messaoud, H. (2013) 'Decentralized observers for a class of large-scale singular systems via LMI', International Journal of Intelligent Computing and Cybernetics, Vol. 6, No. 2, pp.158-181.

Matthews, G.P. and Decarlo, R.A. (1988) 'Decentralized tracking for a class of interconnected nonlinear systems using variable structure control', Automatica, Vol. 24, No. 2, pp.187-193.

Mehraeen, S., Jagannathan, S. and Crow, M. (2011) 'Decentralized dynamic surface control of large-scale interconnected systems in strict-feedback form using neural networks with asymptotic stabilization', IEEE Trans. on Neural Networks, Vol. 22, No. 11, pp.1709-1722.

Mohadjer, M. and Johnson, C.D. (1983) 'Power system control with disturbance accommodation', in Proc. of 22nd IEEE Conf. on Decision and Control, pp.1429-1433, San Antonio, USA.

Nekoukar, V. and Erfanian, A. (2012) 'A decentralized modular control framework for robust control of FES-activated walker-assisted paraplegic walking using terminal sliding mode and fuzzy logic control', IEEE Trans. on Boimedical Engineering, Vol. 59, No. 10, pp.2818-2827.

Panagi, P. and Polycarpou, M. (2011) 'Decentralized fault tolerant control of a class of interconnected nonlinear systems', IEEE Trans. on Automat. Control, Vol. 56, No. 1, pp.178-184. 
Rekik, C., Djemel, M. and Derbel, N. (2007) 'On the suboptimal solution for large scale non-linear control systems using neural networks', Int. J. Modelling, Identification and Control, Vol. 2, No. 4, pp.283-290.

Rezaee, H. and Abdollahi, F. (2014) 'A decentralized cooperative control scheme with obstacle avoidance for a team of mobile robots', IEEE Trans. on Industrial Electronics, Vol. 61, No. 1, pp.347-354.

Richter, S., Lefebvre, S. and Decarlo, R. (1981) 'Control of a class of nonlinear systems by decentralized control', in Proc. 20th IEEE Conference on Decision and Control including the Symposium on Adaptive Processes, Part 1, Vol. 20, pp.1218-1224.

Sandell, N.R., Varaiya, P., Athans, M. and Safonov, M.G. (1978) 'Survey of decentralized control methods for large-scale systems', IEEE Trans. on Automat. Control, Vol. 23, No. 2, pp. $108-128$.

Sato, T., Kano, T. and Ishiguro, A. (2012) 'A decentralized control scheme for an effective coordination of phasic and tonic control in a snake-like robot', Bioinspiration and Biomimetics, Vol. 7, No. 1, pp.1-9.

Shi, L. and Singh, S.K. (1992) 'Decentralized adaptive controller design for large scale systems with higher order interconnections', IEEE Trans. on Automat. Control, Vol. 37, No. 8, pp.1106-1118.

Siljak, D. (1991) Decentralized Control of Complex Systems, Academic Press Inc., London.

Sundareshan, M.K. and Elbanna, R.M. (1991) 'Qualitative analysis and decentralized controller synthesis for a class of large-scale systems with symmetrically interconnected subsystems', Automatica, Vol. 27, No. 2, pp.383-388.

Syrmos, V.L., Abdallah, C.T., Dorato, P. and Grigoriadis, K. (1997) 'Static output feedback - a survey', Automatica, Vol. 33, No. 2, pp.125-137.

Tong, S., Li, Y. and Zhang, H. (2011) 'Adaptive neural network decentralized backstepping output-feedback control for nonlinear large-scale systems with time delays', IEEE Trans. on Neural Networks, Vol. 22, No. 7, pp.1073-1086.

Tong, S., Liu, C. and Li, Y. (2010) 'Fuzzy-adaptive decentralized output-feedback control for large-scale nonlinear systems with dynamical uncertainties', IEEE Trans. on Fuzzy Systems, Vol. 18, No. 5, pp.845-861.

Utkin, V.I. (1992) Sliding Modes in Control Optimization, Springer-Verlag, Berlin.

Vukabratovic, M. and Stokic, D. (1982) Control of Manipulation Robots: Theory and Application, Springer, Berlin

Vukobratovic, M.K. and Stojic, R.D. (1986) 'A decentralized approach to integrated flight control synthesis', Automatica, Vol. 22, No. 6, pp.695-704.

Wang, C. and Soh, Y.C. (1997) 'Decentralized adaptive control using integrator backstepping', Automatica, Vol. 33, No. 9, pp.1719-1724.

Wang, Y., Fan, Y., Wang, Q. and Zhang, Y. (2012) 'Stabilization and synchronization of complex dynamical networks with different dynamics of nodes via decentralized controllers', IEEE Trans. on Circuits and Systems (I), Vol. 59, No. 8, pp.1786-1795.

Wang, Y., Guo, G. and Hill, D.J. (1997) 'Robust decentralized nonlinear controller design for multimachine power systems', Automatica, Vol. 33, No. 9, pp.1725-1733.
Wang, Y. and Zhang, Q. (2001) 'Robust fuzzy decentralized control for nonlinear interconnected descriptor systems', in Proceedings of the 10th IEEE International Conference on Fuzzy Systems, pp.1392-1395, Melbourne, Australia.

Wang, Y. and Zhang, S. (2000) 'Robust control for nonlinear similar composite systems with uncertain parameters', IEE Proc. Part D: Control Theory Appl., Vol. 147, No. 1, pp.80-86.

Wen, C. (1994) 'Decentralized adaptive regulation', IEEE Trans. on Automat. Control, Vol. 39, No. 10, pp.2163-2166.

Wen, C. and Soh, Y. (1999) 'Decentralized model reference adaptive control without restriction on subsystem relative degrees', IEEE Trans. on Automat. Control, Vol. 44, No. 7, pp.1464-1469.

Wen, C., Zhou, J. and Wang, W. (2009) 'Decentralized adaptive backstepping stabilization of interconnected systems with dynamic input and output interactions', Automatica, Vol. 45, No. 1, pp.55-67.

Wo, S., Zou, Y., Sheng, M. and Xu, S. (2007) 'Robust control for discrete-time singular large-scale systems with parameter uncertainty', Journal of the Franklin Institute, Vol. 344, No. 2, pp.97-106

Wu, F., Zhang, X., Ju, P. and Sterling, M. (2008) 'Decentralized nonlinear control of wind turbine with doubly fed induction generator', IEEE Trans. on Power Systems, Vol. 23, No. 2, pp.613-621.

Wu, H. (2002) 'Decentralized adaptive robust control for a class of large-scale systems including delayed state perturbations in the interconnections', IEEE Trans. on Automat. Control, Vol. 47, No. 10, pp.1745-1751.

Wu, Y., Cao, X. and Zheng, P. (2012) 'Variable structure-based decentralized relative attitude-coordinated control for satellite formation', IEEE Aerospace and Electronic Systems Magazine, Vol. 27, No. 12, pp.18-25.

Xie, S. and Xie, L. (2000) 'Decentralized stabilization of a class of interconnected stochastic nonlinear systems', IEEE Trans. on Automat. Control, Vol. 45, No. 1, pp.132-137.

$\mathrm{Xu}, \mathrm{X} ., \mathrm{Wu}, \mathrm{Y}$. and Huang, W. (1990) 'Variable-structure control approach of decentralised model-reference adaptive systems', IEE Proc. Part D: Control Theory Appl., Vol. 137, No. 5, pp.302-306.

Yan, X.G. (1996) Robust Structural Holographic Control for Complex Large-scale Similar Interconnected Systems, $\mathrm{PhD}$ Thesis: Northeastern University, Shenyang, China (in Chinese).

Yan, X.G., Chen, I.M. and Lam, J. (2001) 'D-type learning control for nonlinear time-varying systems with unknown initial states and inputs', Transactions of the Institute of Measurement and Control, Vol. 23, No. 2, pp.101-114.

Yan, X.G. and Dai, G.Z. (1998) 'Decentralized output feedback robust control for nonlinear large-scale systems', Automatica, Vol. 34, No. 11, pp.1469-1472.

Yan, X.G. and Edwards, C. (2008) 'Robust decentralized actuator fault detection and estimation for large-scale systems using a sliding mode observer', Int. J. Control, Vol. 81, No. 4 pp.591-606.

Yan, X.G., Edwards, C. and Spurgeon, S.K. (2004) 'Decentralised robust sliding mode control for a class of nonlinear interconnected systems by static output feedback', Automatica, Vol. 40, No. 4, pp.613-620. 
Yan, X.G., Edwards, C. and Spurgeon, S.K. (2009) 'Global time-delay dependent decentralized sliding mode control using only output information', in Proceedings of the Joint Conference of 48th IEEE CDC and 28th Chinese Control Conference, pp.6709-6715, Shanghai, China.

Yan, X.G., Edwards, C., Spurgeon, S.K. and Bleijs, J.A.M. (2004) 'Decentralised sliding mode control for multimachine power systems using only output information', IEE Proc. Part D: Control Theory Appl., Vol. 151, No. 5, pp.627-631.

Yan, X.G., Gao, L.Q. and Zhang, S.Y. (1997) 'Holographic robust control for nonlinear similar composite systems with uncertain parameters', Acta Automat. Sinica, Vol. 23, No. 5, pp.654-659.

Yan, X.G., Lam, J. and Dai, G.Z. (1999) 'Decentralized robust control for nonlinear similar large-scale systems', Computer and Electrical Engineering, Vol. 25, No. 3, pp.169-179.

Yan, X.G., Lam, J., Li, H.S. and Chen, I.M. (2000) 'Decentralized control of nonlinear large-scale systems using dynamic output feedback', J. Optim. Theory Appl., Vol. 104, No. 2, pp.459-475.

Yan, X.G., Lam, J. and Xie, L. (2003) 'Robust observer deisgn for non-linear interconnected systems using structural characteristics', Int. J. Control, Vol. 76, No. 7, pp.741-746.

Yan, X.G., Spurgeon, S.K. and Edwards, C. (2006) 'Decentralised sliding mode control for nonminimum phase interconnected systems based on a reduced-order compensator', Automatica, Vol. 42, No. 10, pp.1821-128.

Yan, X.G., Spurgeon, S.K. and Edwards, C. (2010) 'Static output feedback sliding mode control for time-varying delay systems with time-delayed nonlinear disturbances', Int. J. Robust Nonlinear Control, Vol. 20, No. 7, pp.777-788.

Yan, X.G., Spurgeon, S.K. and Edwards, C. (2012) 'Global decentralised static output feedback sliding mode control for interconnected time-delay systems', IET Control Theory and Applications, Vol. 6, No. 2, pp.192-202.

Yan, X.G., Spurgeon, S.K. and Edwards, C. (2013) 'Decentralised stabilisation for nonlinear time delay interconnected systems using static output feedback', Automatica, Vol. 49, No. 2, pp.633-641.

Yan, X. G., Wang, J., Lü, X. and Zhang, S. (1998) 'Decentralized output feedback robust stabilization for a class of nonlinear interconnected systems with similarity', IEEE Trans. on Automat. Control, Vol. 43, No. 2, pp.294-299.

Yan, X.G. and Xie, L. (2003) 'Reduced-order control for a class of nonlinear similar interconnected systems with mismatched uncertainty', Automatica, Vol. 39, No. 1, pp.91-99.

Yang, G.H. and Zhang, S.Y. (1995) 'Stabilizing controllers for uncertain symmetric composite systems', Automatica, Vol. 31, No. 2, pp.337-340.

Yang, G.H. and Zhang, S.Y. (1996) 'Decentralized control of a class of large-scale systems with symmetrically interconnected subsystems', IEEE Trans. on Automat. Control, Vol. 41, No. 5, pp.710-713.

Yang, T-C., Cimen, H. and Zhu, Q. (1998) 'Decentralised load-frequency controller design based on structured singular values', IEE Proc. Part D: Control Theory Appl., Vol. 145, No. 1, pp.7-14.

Yang, Z.J., Fukushima, Y. and Qin, P. (2012) 'Decentralized adaptive robust control of robot manipulators using disturbance observers', IEEE Trans. on Control Systems Technology, Vol. 20, No. 5, pp.1357-1365.
Yasuda, K. and Noso, F. (1996) 'Decentralized quadratic stabilization of interconnected descriptor systems', in Proceedings of 35th IEEE Conference on Decision and Control, pp.4264-4269, Florida, USA.

Ye, X. (1999) 'Decentralized adaptive regulation with unknown high-frequency-gain signs', IEEE Trans. on Automat. Control, Vol. 44, No. 11, pp.2072-2076.

Yoo, S., Park, J. and Choi, Y. (2009) 'Decentralized adaptive stabilization of interconnected nonlinear systems with unknown non-symmetric dead-zone inputs', Automatica, Vol. 45, No. 2, pp.436-443.

Yu, R. (2005) 'Regularizability of linear time-invariant descriptor systems under decentralized control', Automatica, Vol. 41, No. 9, pp.1639-1644.

Yu, R. and Wang, D. (2005) 'On impulsive modes of linear singular systems subject to decentralized output feedback', IEEE Trans. on Automat. Control, Vol. 48, No. 10, pp.1804-1809.

Zak, S.H. and Hui, S. (1993) 'Output feedback variable structure controllers and state estimators for uncertain/nonlinear dynamic systems', IEE Proc. Part D: Control Theory Appl., Vol. 140, No. 1, pp.41-50.

Zhai, G.S., Yoshida, M., Imae, J. and Kobayashi, T. (2006) 'Decentralized $\mathrm{H}_{2}$ controller design for descriptor systems: an LMI approach', Nonlinear Dynamics and Systems Theory, Vol. 6, No. 1, pp.99-109.

Zhang, Q. (1997) Decentralized and Robust Control for Large-scale Descriptor Systems, Northwest Polytechnic University Press, Xi'an.

Zhang, Q.L. (1990) 'On generalized decentralized fixed modes in descriptor systems', Systems and Control Letters, Vol. 15, No. 4, pp.295-301.

Zhang, X., Liu, L., Feng, G. and Zhang, C. (2013) 'Output feedback control of large-scale nonlinear time-delay systems in lower triangular form', Automatica, Vol. 49, No. 11, pp.3476-3483.

Zhang, Y., Li, S. and Zhu, Q. (2007) 'Backstepping-enhanced decentralised PID control for MIMO processes with an experimental study', IET Control Theory and Applications, Vol. 1, No. 3, pp.704-712.

Zhou, J. (2008) 'Decentralized adaptive control for large-scale time-delay systems with dead-zone input', Automatica, Vol. 44, No. 7, pp.1790-99.

Zhou, J. and Wen, C. (2008a) Adaptive Backstepping Control of Uncertain Systems: Nonsmooth Nonlinearities, Interactions or Time-Variations, Lecture Notes in Control and Information Sciences, Vol. 372, Springer-Verlagm Berlin Heidelberg.

Zhou, J. and Wen, C. (2008b) 'Decentralized backstepping adaptive output tracking of interconnected nonlinear systems', IEEE Trans. on Automat. Control, Vol. 53, No. 10, pp.2378-2384.

Zhou, Q., Shi, P., Liu, H. and Xu, S. (2012) 'Neural-network-based decentralized adaptive output-feedback control for large-scale stochastic nonlinear systems', IEEE Trans. on Systems, Man and Cybernetics, Vol. 42, No. 6, pp.1608-1619. 